\title{
Full Proportionate Functional Link Adaptive Filters for Nonlinear Acoustic Echo Cancellation
}

\author{
Danilo Comminiello*, Michele Scarpiniti*, Luis A. Azpicueta-Ruiz ${ }^{\dagger}$, Jerónimo Arenas-García, ${ }^{\dagger}$ and Aurelio Uncini* \\ *Department of Information Engineering, Electronics and Telecommunications, "Sapienza" University of Rome, Italy \\ Email: \{danilo.comminiello, michele.scarpiniti, aurelio.uncini\}@uniroma1.it \\ †Department of Signal Theory and Communications, Universidad Carlos III de Madrid, Spain \\ Email: \{lazpicueta, jarenas\}@ tsc.uc3m.es
}

\begin{abstract}
Nonlinear acoustic echo cancellation (NAEC) can be mainly addressed by solving two different sub-problems: the estimation of the acoustic impulse response and the modeling of the nonlinearities rebounding in it, mostly caused by the electroacoustic chain. Both the modeling processes share an important characteristic: the majority of the parameters to be estimated are very close to zero, with only a small fraction of them having non-negligible magnitude. In this paper, a novel NAEC model is proposed taking into account both the above sub-problems under a joint optimization problem. In particular, the proposed model involves two separate and parallel filters, one mainly focusing on the estimation of the acoustic impulse response (AIR) and the other one aiming at the nonlinear modeling. In order to optimize the modeling processes, both the filters are adapted by using a joint proportionate algorithm. Experimental results prove the effectiveness of the proposed model in NAEC problems.
\end{abstract}

\section{INTRODUCTION}

The presence of nonlinear acoustic echo has always been considered a difficult problem to be solved and it still represents a challenging research topic, especially because of the widespread use of commercial hands-free speech communication systems. Nonlinearities may be produced by different sources, ranging from loudspeakers, to vibrations of device materials, such as plastic enclosures or displays of mobile devices [1]. Each different device may generate a certain nonlinearity, whose level may also vary in time. Moreover, large speech signal peaks may be strongly corrupted. This makes online modeling of nonlinearity a quite difficult task.

In the recent years, several NAEC models have been proposed. State-space based models have received increasing attention, such as those focusing on Bayesian learning [2], [3] or on the elitist particle filter [4]. In [5], the NAEC approach is based on the representation of the physical model of the loudspeaker. Other NAEC models have been expressly designed for mobile devices [6]-[8]. Even a hardware solution based on voltage and current feedback values has been proposed [9]. However, most of the NAEC models introduced in the last years are based on particular classes of linear-in-theparameters (LIP) nonlinear filters, including recent advances on Volterra filters [10], [11], kernel adaptive filters [12], [13], Hammerstein spline adaptive filters [14], or parallel Hammerstein architectures [15]. In this work, a new NAEC method based on LIP nonlinear filters is proposed. In particular, we consider the class of functional link adaptive filters (FLAFs). The FLAF was firstly introduced as an effective method for nonlinear modeling [1], [16], [17]. The split FLAF (SFLAF) scheme was proposed to better exploit the capabilities of linear and nonlinear filtering in parallel. While in [1], [16] the adaptive algorithms for the linear and nonlinear branches were chosen independently of each other, here we aim at providing an optimized learning algorithm involving both the linear and the nonlinear adaptations.

In order to derive the joint learning algorithm, we take into account some considerations on both the linear and nonlinear modeling. In NAEC, both in the estimation of the acoustic echo path and in the modeling of the nonlinearities, an unknown response is composed of a few number of coefficients with large magnitude, but most of them have a value very close to zero. This permits to obtain performance improvement when adopting any regularized learning solution. In particular, the estimation of the AIR has been proved to be robust to disturbing signals when regularization is taken into account in the least-square solution [18]-[22]. On the other hand, in the nonlinear modeling, the expansion of the input signal may also show some sparse behavior, and this has encouraged the use of proportionate algorithms even for LIP nonlinear filters [23][27]. Therefore, in this work, we propose an SFLAF model for NAEC that is capable of exploiting the capabilities of both the linear and nonlinear modeling, by providing a general formulation for the joint optimization problem involving both the estimation processes.

The paper is organized as follows. In Section II, the SFLAF architecture is briefly introduced. The proposed full proportionate algorithm for the SFLAF scheme is derived in Section III. In Section IV, experiments show the effective results of the proposed method, and, in the end, in Section V our conclusions are drawn.

\section{The Split Functional Link AdAptive Filter}

The output of a real-world system to be identified is very often generated by any combination of linear and nonlinear elements, as in the case of online applications like NAEC. For this reason, an appropriate choice for the modeling of realworld systems is to adopt an architecture based on the split functional link adaptive filter (SFLAF). The SFLAF scheme 


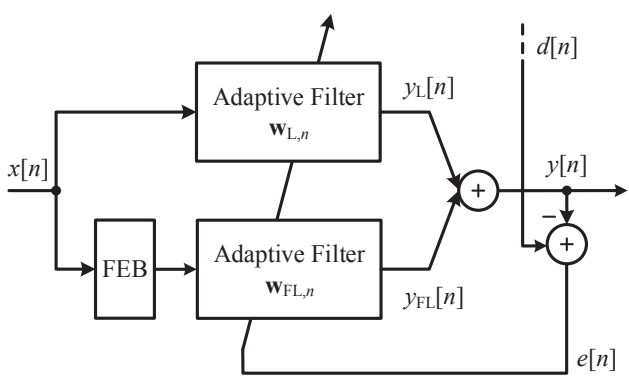

Fig. 1. The SFLAF architecture adopted for the proposed algorithm.

[1], represented in Fig. 1, is composed of a linear branch in parallel with a nonlinear one. The linear branch simply involves an adaptive filter, aiming at modeling the linear part of an unknown system response. On the other hand, the nonlinear branch can focus on the nonlinear modeling, without any need to estimate the linear components of the AIR. In particular, the nonlinear branch is an LIP nonlinear filter, composed of a functional expansion block (FEB) and an adaptive filter.

Let us consider the input sample $x[n]$ to the unknown system. This is collected in a linear input buffer $\mathbf{x}_{\mathrm{L}, n} \in$ $\mathbb{R}^{M}=\left[\begin{array}{llll}x[n] & x[n-1] & \ldots & x[n-M+1\end{array}\right]^{\mathrm{T}}$, where $M$ is the buffer length, i.e., the length of the coefficient vector $\mathbf{w}_{\mathrm{L}, n} \in \mathbb{R}^{M}=\left[\begin{array}{llll}w_{\mathrm{L}, 0}[n] & w_{\mathrm{L}, 1}[n] & \ldots & w_{\mathrm{L}, M-1}[n]\end{array}\right]^{\mathrm{T}}$. Then, the linear output is the result of the filtering $y_{\mathrm{L}}[n]=$ $\mathbf{x}_{\mathrm{L}, n}^{\mathrm{T}} \mathbf{w}_{\mathrm{L}, n-1}$. The input sample $x[n]$ is also received by the nonlinear branch and collected in another buffer $\mathbf{x}_{\mathrm{FL}, n} \in$ $\mathbb{R}^{M_{\mathrm{i}}}=\left[\begin{array}{llll}x[n] & x[n-1] & \ldots & x\left[n-M_{\mathrm{i}}+1\right.\end{array}\right]^{\mathrm{T}}$, where $M_{\mathrm{i}}$ represents the length of $\mathrm{x}_{\mathrm{FL}, n}$. This signal is processed by the FEB, which is a nonlinear block containing a series of functions, called functional links, satisfying universal approximation properties. The set of functional links is denoted as $\Phi=\left\{\varphi_{0}(\cdot), \varphi_{1}(\cdot), \ldots, \varphi_{Q_{\mathrm{f}}-1}(\cdot)\right\}$, where $Q_{\mathrm{f}}$ is the number of different functional links contained in the set. The $i$-th element of $\mathbf{x}_{\mathrm{FL}, n}$ is expanded by the functional links of $\Phi$, thus producing a vector $\overline{\mathbf{g}}_{i, n} \in \mathbb{R}^{Q_{\mathrm{f}}}$ :

$$
\overline{\mathbf{g}}_{i, n}=\left[\begin{array}{c}
\varphi_{\mathrm{o}}(x[n-i]) \\
\varphi_{1}(x[n-i]) \\
\vdots \\
\varphi_{Q_{\mathrm{f}}-1}(x[n-i])
\end{array}\right] .
$$

The concatenation of $\overline{\mathbf{g}}_{i, n}$, for $i=0, \ldots, M_{\mathrm{i}}-1$, yields the expanded buffer $\mathbf{g}_{n} \in \mathbb{R}^{M_{\mathrm{e}}}$ :

$$
\begin{aligned}
\mathbf{g}_{n} & =\left[\begin{array}{llll}
\mathbf{g}_{0, n}^{\mathrm{T}} & \mathbf{g}_{1, n}^{\mathrm{T}} & \ldots & \mathbf{g}_{M_{\mathrm{i}}-1, n}^{\mathrm{T}}
\end{array}\right]^{\mathrm{T}} \\
& =\left[\begin{array}{llll}
g_{0}[n] & g_{1}[n] & \ldots & g_{M_{\mathrm{e}}-1}[n]
\end{array}\right]^{\mathrm{T}} .
\end{aligned}
$$

In (2), $M_{\mathrm{e}} \geq M_{\mathrm{i}}$ is the length of the expanded buffer. It is worth noting that $M_{\mathrm{e}}=M_{\mathrm{i}}$ if $Q_{\mathrm{f}}=1$.

Functional links may be derived by a large variety of nonlinear expansions. In this work, the functional links of the FEB are generated by a nonlinear trigonometric series expansion, i.e.:

$$
\varphi_{j}(x[n-i])= \begin{cases}\sin (p \pi x[n-i]), & j=2 p-2 \\ \cos (p \pi x[n-i]), & j=2 p-1\end{cases}
$$

in which $p=1, \ldots, P$ is the expansion index, $P$ is the expansion order, and $j=0, \ldots, Q_{\mathrm{f}}-1$ is the functional link index. It is easy to verify that, in this case, the functional link set $\Phi$ contains $Q_{\mathrm{f}}=2 P$ elements.

In the described model of the nonlinear branch, the linear part of a system to be identified can be modeled by a separate filter and therefore the expanded vector $\mathbf{g}_{n}$ contains nonlinear elements only, as we describe in the next subsection. The trigonometric expansion in (3) is memoryless, since there are no cross-products with past samples. However, the trigonometric expansion can be easily extended to include any memory (see [1] for a detailed explanation).

The resulting vector $\mathbf{g}_{n}$ is filtered by $\mathbf{w}_{\mathrm{FL}, n} \in \mathbb{R}^{M_{\mathrm{e}}}=$

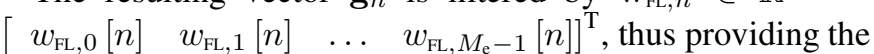
nonlinear output $y_{\mathrm{FL}}[n]=\mathbf{g}_{n}^{\mathrm{T}} \mathbf{w}_{\mathrm{FL}, n-1}$. The overall SFLAF output signal is obtained by summing the individual branch outputs, i.e.:

$$
y[n]=y_{\mathrm{L}}[n]+y_{\mathrm{FL}}[n] .
$$

As a consequence, the error signal, which is used for the adaptation of the two filters, can be achieved as $e[n]=d[n]-y[n]$. The filter updates can be performed by adopting any adaptive algorithm (see for example [28], [29]), including proportionate algorithms that have been widely employed in AEC [20], [30][32]. In particular, in the next section we derive a regularized algorithm for SFLAF that involves proportionate adaptation in both the branches; we refer to such SFLAF as "full proportionate".

\section{Full ProportionAte SFLAF}

In NAEC problems, the modeling of the linear part of a system is required mainly to estimate the AIR. However, a nonlinear modeling is also required to tackle the distortions introduced in the echo signal by the unknown system. An AIR is known to show often some sparse characteristics [32], because the most significant part of it is contained in its head, containing both the direct path and the early reflections of an acoustic space. On the other hand, since the degree and type of a nonlinearity is not known a priori, it is reasonable to overestimate the number of functional links, so that the optimal solution for $\mathbf{w}_{\mathrm{FL}, n}$ can be highly sparse. In both the linear and nonlinear modeling, a performance improvement might be achieved by considering a regularized learning solution. To this end, a "full" regularized adaptive algorithm can be developed for the SFLAF scheme to give higher importance to the filter coefficients showing a significant performance contribution in both linear and nonlinear modeling.

\section{A. Joint derivation of the full PSFLAF}

The update equations of the two filters $\mathbf{w}_{\mathrm{L}, n}$ and $\mathbf{w}_{\mathrm{FL}, n}$ can be achieved by a joint derivation. The optimization procedure involves a formulation similar to [23], but with a novel resulting derivation due to the full weighted mask. Let us define the following joint input and weight vectors, respectively: 


$$
\begin{aligned}
\mathbf{x}_{n} \in \mathbb{R}^{M+M_{\mathrm{e}}} & =\left[\begin{array}{c}
\mathbf{x}_{\mathrm{L}, n} \\
\mathbf{g}_{n}
\end{array}\right] \\
\mathbf{w}_{n} \in \mathbb{R}^{M+M_{\mathrm{e}}} & =\left[\begin{array}{c}
\mathbf{w}_{\mathrm{L}, n} \\
\mathbf{w}_{\mathrm{FL}, n}
\end{array}\right] .
\end{aligned}
$$

The output signal of the full PSFLAF can be now obtained as $y[n]=\mathbf{x}_{n}^{\mathrm{T}} \mathbf{w}_{n-1}$, which is equivalent to (4).

We can denote the following difference vector:

$$
\widetilde{\mathbf{w}}_{n}=\mathbf{w}_{n}-\mathbf{w}_{n-1} .
$$

Taking into account the least-perturbation property and the natural gradient adaptation, as suggested in [23], it is possible to express the constrained optimization problem as:

$$
\begin{gathered}
\arg \min _{\mathbf{w}_{n}}\left\|\widetilde{\mathbf{w}}_{n}\right\|_{\mathbf{Q}_{n}^{-1}}^{2} \\
\text { s.t. } \quad \varepsilon[n]=0
\end{gathered}
$$

where $\mathbf{Q}_{n}^{-1}$ is a distance correction matrix with respect to the Euclidean metric, and the constraint $\varepsilon[n]=0$ can be derived from the a posteriori output estimation error signal:

$$
\varepsilon[n]=d[n]-\mathbf{x}_{n}^{\mathrm{T}} \mathbf{w}_{n} .
$$

The problem in (8) can be solved by deriving the Lagrangian function:

$$
\mathcal{L}[n]=\widetilde{\mathbf{w}}_{n}^{\mathrm{T}} \mathbf{Q}_{n}^{-1} \widetilde{\mathbf{w}}_{n}+\lambda\left(d[n]-\mathbf{x}_{n}^{\mathrm{T}} \mathbf{w}_{n}\right)
$$

where $\lambda$ is a Lagrange multiplier. Taking the gradient of (10) with respect to $\mathbf{w}_{n}$ and then setting the result to zero yelds:

$$
\nabla_{\mathbf{w}} \mathcal{L}[n]=2 \mathbf{Q}_{n}^{-1} \widetilde{\mathbf{w}}_{n}-\lambda \mathbf{x}_{n}=0,
$$

which can be written as

$$
\mathbf{w}_{n}=\mathbf{w}_{n-1}+\frac{1}{2} \lambda \mathbf{Q}_{n} \mathbf{x}_{n} .
$$

In order to solve the Lagrange multiplier $\lambda$ both sides of (12) can be left-multiplied by $\mathbf{x}_{n}^{\mathrm{T}}$ :

$$
\mathbf{x}_{n}^{\mathrm{T}} \mathbf{w}_{n}-\mathbf{x}_{n}^{\mathrm{T}} \mathbf{w}_{n-1}=\frac{1}{2} \lambda \mathbf{x}_{n}^{\mathrm{T}} \mathbf{Q}_{n} \mathbf{x}_{n}
$$

The left side of (13) can be rewritten by taking into account that $\mathbf{x}_{n}^{\mathrm{T}} \mathbf{w}_{n}=d[n]$, due to the constraint in (8), and $d[n]-$ $\mathbf{x}_{n}^{\mathrm{T}} \mathbf{w}_{n-1}=e[n]$. Hence:

$$
\lambda=\frac{2 e[n]}{\mathbf{x}_{n}^{\mathrm{T}} \mathbf{Q}_{n} \mathbf{x}_{n}} .
$$

Replacing (14) in (12), we obtain:

$$
\mathbf{w}_{n}=\mathbf{w}_{n-1}+\Lambda \frac{\mathbf{Q}_{n} \mathbf{x}_{n}}{\mathbf{x}_{n}^{\mathrm{T}} \mathbf{Q}_{n} \mathbf{x}_{n}+\delta} e[n],
$$

which represents the equation of the updating rule for the joint coefficient vector of the full PSFLAF. In (15), two parameters have been added to ensure a correct adaptation [28], [29]: the regularization factor $\delta$ and a diagonal matrix of the step-size parameters $\boldsymbol{\Lambda}=\operatorname{diag}\left\{\begin{array}{ccc}\mu_{0} & \ldots & \mu_{M+M_{\mathrm{e}}-1}\end{array}\right\}$. In particular, we choose the elements of $\boldsymbol{\Lambda}$ as $\mu_{k}=\mu_{\mathrm{L}}$, for $k=0, \ldots, M-$ 1 , and $\mu_{k}=\mu_{\mathrm{FL}}$, for $k=M, \ldots, M+M_{\mathrm{e}}-1$, in order to preserve the flexibility of the split scheme to choose different parameter settings for the coefficients of the filters on the linear and nonlinear branches [1].

\section{B. Derivation of the proportionate matrix}

The proportionate matrix $\mathbf{Q}_{n}$ in (15) aims at weighting the coefficients of $\mathbf{w}_{n}$ in a proportional fashion with respect to the contribution they give to the linear and nonlinear modeling. We choose the weighting matrix $\mathbf{Q}_{n}$ as a diagonal matrix that can be expressed as:

$$
\mathbf{Q}_{n} \in \mathbb{R}^{M+M_{\mathrm{e}}}=\operatorname{diag}\left\{q_{0}[n] \quad \ldots \quad q_{M+M_{\mathrm{e}}-1}[n]\right\} .
$$

The diagonal elements of $\mathbf{Q}_{n}$ are derived by using the filter coefficients computed at the time instant $n-1$. In particular, the larger the magnitude of a coefficient of $\mathbf{w}_{n-1}$, the higher the corresponding weighting. As a consequence, the most significant coefficients benefits from a faster adaptation. This asymmetric weighting will make the filter converge faster in situations where the optimal solution is sparse (or quasisparse), in contrast with the original SFLAF, which has a uniform weighting for all the functional links.

The elements of the proportionate matrix (16) are derived from the improved proportionate normalized least mean square (IPNLMS) algorithm [30] with application to the proposed SFLAF model, thus yielding:

$$
q_{k}[n]=\left\{\begin{array}{l}
\frac{1-\alpha_{\mathrm{L}}}{2 M}+\left(1+\alpha_{\mathrm{L}}\right) \frac{\left|w_{k}[n-1]\right|}{\xi+2\left\|\mathbf{w}_{n-1}\right\|_{1}}, \\
k=0, \ldots, M-1 \\
\frac{1-\alpha_{\mathrm{FL}}}{2 M_{\mathrm{e}}}+\left(1+\alpha_{\mathrm{FL}}\right) \frac{\left|w_{k}[n-1]\right|}{\xi+2\left\|\mathbf{w}_{n-1}\right\|_{1}}, \\
k=M, \ldots, M+M_{\mathrm{e}}-1
\end{array}\right.
$$

where the scalar $\xi$ is a small positive value avoiding divisions by zero. In (17), the proportionality factors $-1 \leq \alpha_{\mathrm{L}}, \alpha_{\mathrm{FL}} \leq 1$ have the task of balancing the proportionality. In fact, when they assume a value close to 1 a high degree of sparseness is expected both for the functional link expansion and for the linear branch, while, on the contrary, a low degree is expected when the proportionality factors are close to -1 , thus reducing in the limit the adaptation to a normalized least mean square (NLMS) algorithm. It is worth noting that even for the proportionality factors it is possible to choose two different values, $\alpha_{\mathrm{L}}$ and $\alpha_{\mathrm{FL}}$, respectively for the linear and the nonlinear branches to preserve the flexibility of the SFLAF architecture.

\section{EXPERIMENTAL RESULTS}

The proposed full PSFLAF is assessed in a simulated NAEC scenario [1]. In particular, as represented in Fig. 3, the unknwon system to identify is a cascade of a nonlinear block, which introduces a loudspeaker distortion, followed by a linear one that is the echo path between the loudspeaker and the microphone. The sparse AIR is achieved by simulation considering reverberation time of $T_{60}=150 \mathrm{~ms}$, sampling frequency of $8 \mathrm{kHz}$ and length $M=1200$ samples, and it is depicted in Fig. 2. In order to reproduce an asymmetric loudspeaker distortion, the input signal $x[n]$ is processed by a memoryless sigmoidal nonlinearity [1]:

$$
\bar{y}[n]=\gamma\left(\frac{1}{1+e^{(-\rho r[n])}}-\frac{1}{2}\right)
$$




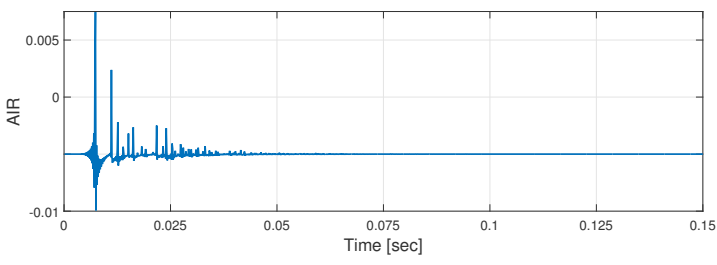

Fig. 2. Acoustic impulse response used in NAEC scenarios.

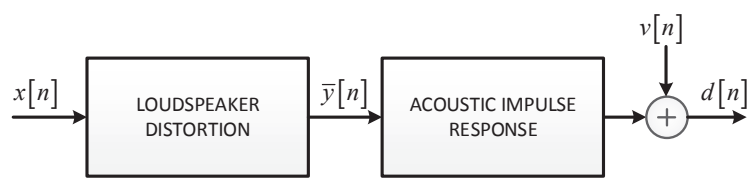

Fig. 3. Scheme of the NAEC system to be identified.

with:

$$
r[n]=\frac{3}{2} x[n]-\frac{3}{10} x^{2}[n] .
$$

In (18), the parameter $\gamma$ represents the sigmoid gain and its value is set to $\gamma=2$. The sigmoid slope is represented by $\rho$ :

$$
\rho=\left\{\begin{array}{ll}
4, & r[n]>0 \\
\frac{1}{2}, & r[n] \leq 0
\end{array} .\right.
$$

The signal $\bar{y}[n]$ obtained by (18) is then convolved by the AIR. Additive Gaussian noise $v[n]$ is present at the microphone location providing $30 \mathrm{~dB}$ of signal-to-noise ratio (SNR). Therefore, the desired signal is obtained as $d[n]=\bar{y}[n]+v[n]$. Two kinds of signals are used as input $x[n]$ : colored noise and female speech input. The colored noise signal is generated by means of a first-order autoregressive model, with transfer function $\sqrt{1-\theta^{2}} /\left(1-\theta z^{-1}\right)$, where $\theta=0.8$. The length of both the experiments is 10 seconds. An abrupt change in the AIR is also introduced at half of the experiment length, by a right shift of the AIR of 20 samples. In the same instant, an abrupt change of the nonlinearity is also introduced by setting $\rho=5 / 2$ for $r[n] \leq 0$ in eq. (20).

Performance results are evaluated according to the echo return loss enhancement (ERLE), which is defined in $\mathrm{dB}$ as:

$$
\operatorname{ERLE}[n]=10 \log _{10}\left(\frac{\mathrm{E}\left\{d^{2}[n]\right\}}{\mathrm{E}\left\{e^{2}[n]\right\}}\right),
$$

in which $\mathrm{E}\{\cdot\}$ denotes the mathematical expectation. We compare the performance of the proposed full PSFLAF with those of the NLMS, the IPNLMS, the SFLAF [1], and the PSFLAF presented in [23], which uses a proportionate algorithm only for the adaptive filter on the nonlinear branch. It is worth noting that performance of SFLAF and PSFLAF have been extensively compared with other state-of-the-art methods in [1], [16], [23]. We use the following parameter setting, seeking the best performance of each algorithm: input buffer length $M_{\mathrm{i}}=M / 4$, step sizes $\mu_{\mathrm{L}}=0.1$ and $\mu_{\mathrm{LL}}=0.1$, for all the filters except the full PSFLAF for which we use $\mu_{\mathrm{L}}=1$, $\mu_{\mathrm{FL}}=0.8$. We also choose regularization parameter $\delta=10^{-2}$, proportionality factors $\alpha_{\mathrm{L}}=\alpha_{\mathrm{FL}}=0$, and expansion order

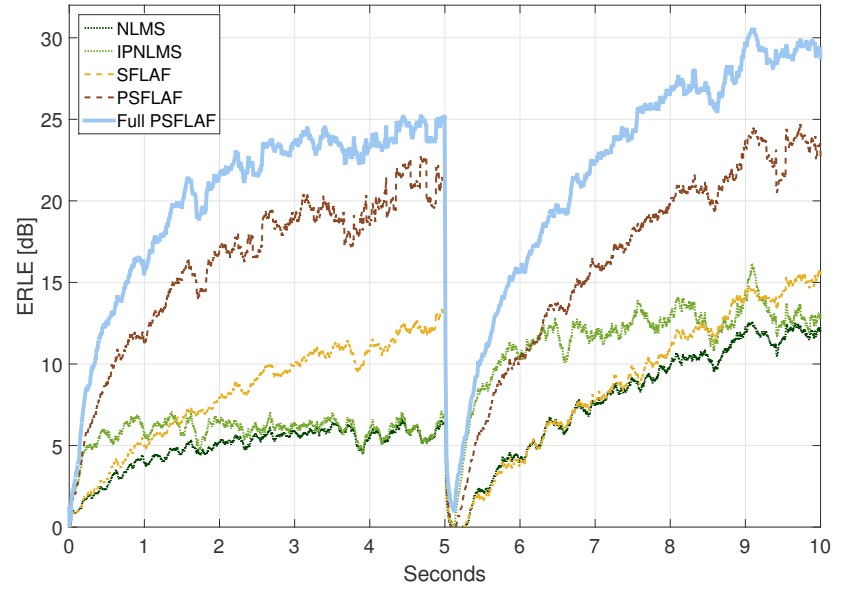

Fig. 4. ERLE evolution in case of colored noise input.

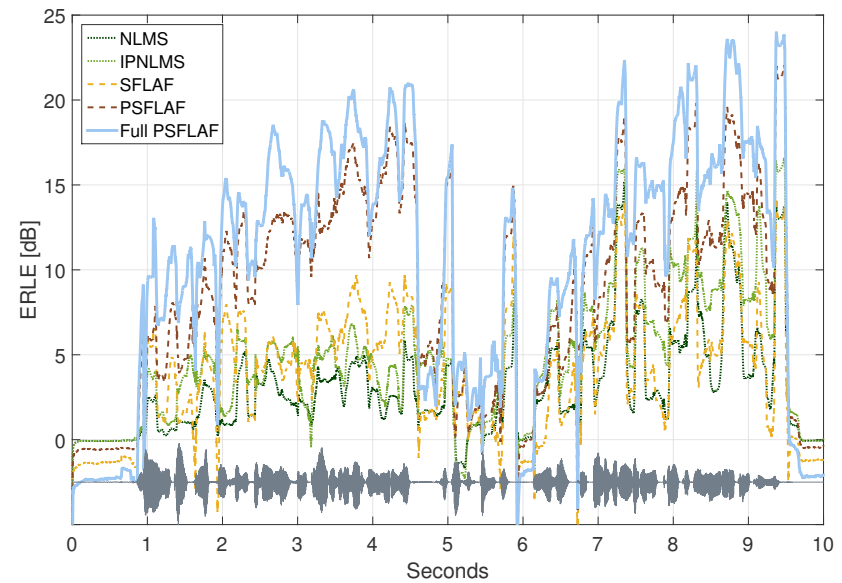

Fig. 5. ERLE evolution in case of female speech input.

$P=10$. All the FLAFs involved are memoryless. For the case of female speech input, we only change $\mu_{\mathrm{L}}=0.01$ and $\mu_{\mathrm{FL}}=0.5$ for all the filters except the full PSFLAF, whose step-size values remain unchanged.

ERLE results for the case of colored input are depicted in Fig. 4, where it can be seen the convergence performance improvement of the full PSFLAF with respect to the other algorithms. It also shows superior tracking performance when changes occur in linear and nonlinear branches. Performance results for the case of speech signal are shown in Fig. 5, where a significant improvement is evident with gains of around 5 $\mathrm{dB}$, especially in proximity of the signal peaks, when the distortions are more easily perceived.

\section{CONCLusions}

In this paper, a novel NAEC system based on functional links has been introduced. The proposed algorithm shows the structure of a split FLAF, involving a linear adaptive filter that aims at the estimation of the AIR, and an LIP nonlinear filter devoted at the modeling of the nonlinearities rebounding in the acoustic path. A joint proportionate learning algorithm has been developed for this structure, giving 
robustness to the modeling of both the AIR and nonlinearities. Experimental results have shown the effectiveness of the full PSFLAF algorithm in providing improved performance in the presence of both colored noise and speech input signals for NAEC problems. Future research will focus on extending the proposed framework to include other proportionate learning algorithms, other LIP nonlinear filters besides the FLAF, and adaptive schemes to manage the value of the proportionate factors in an online manner.

\section{ACKNOWLEDGMENT}

The work of M. Scarpiniti and A. Uncini is partially supported by the Italian National Project "GAUChO - A Green Adaptive Fog Computing and Networking Architecture", under grant number 2015YPXH4W.

The work of L. A. Azpicueta-Ruiz is partially supported by Comunidad de Madrid under grant 'CASI-CAM-CM' (id. S2013/ICE-2845), by the Spanish Ministry of Economy and Competitiveness (under grant DAMA (TIN2015-70308REDT) and grant TEC2014-52289-R), and by the European Union. The work of J. Arenas-García has been partly funded by MINECO project TEC2014-52289-R, and by Comunidad de Madrid project PRICAM S2013/ICE-2933.

\section{REFERENCES}

[1] D. Comminiello, M. Scarpiniti, L. A. Azpicueta-Ruiz, J. Arenas-García, and A. Uncini, "Functional link adaptive filters for nonlinear acoustic echo cancellation," IEEE Trans. Audio, Speech, Language Process., vol. 21, no. 7, pp. 1502-1512, July 2013.

[2] S. Malik, G. Enzner, J.P. Vartiainen, J.P. Sjoberg, and V.M. Myllyla, "Recursive Bayesian controllers for non-linear acoustic echo cancellation and suppression systems," Dec. 30 2014, US Patent 8,924,337.

[3] C. Huemmer, R. Maas, C. Hofmann, and W. Kellermann, "A Bayesian network approach to linear and nonlinear acoustic echo cancellation," EURASIP J. Advances in Signal Process., vol. 98, pp. 1-11, Nov. 2015.

[4] C. Huemmer, C. Hofmann, R. Maas, and W. Kellermann, "The significance-aware EPFES to estimate a memoryless preprocessor for nonlinear acoustic echo cancellation," in IEEE Global Conf. Signal and Information Process. (GlobalSIP), Atlanta, GA, Dec. 2014, pp. 557-561.

[5] S. S. Payal, V. J. Mathews, A. Iyer, R. Lambert, and J. Hutchings, "Equalization of excursion and current-dependent nonlinearities in loudspeakers," in IEEE Int. Conf. Acoust., Speech and Signal Process. (ICASSP), Florence, Italy, May 2014, pp. 4-9.

[6] O. Hoshuyama, "Dual-microphone echo canceller for suppressing loud nonlinear echo," in IEEE Int. Conf. Acoust., Speech and Signal Process. (ICASSP), Kyoto, Japan, Mar. 2012, pp. 181-184.

[7] L. K. Gudupudi, C. Beaugeant, N. Evans, M. Mossi, and L. Lepauloux, "A comparison of different loudspeaker models to empirically estimated non-linearities," in Joint Workshop Hands-free Speech Commun. and Microphone Arrays (HSCMA), 2014.

[8] Y. Huang, J. Skoglund, and A. Luebs, "Bi-magnitude processing framework for nonlinear acoustic echo cancellation on Android devices," in IEEE Int. Workshop on Acoustic Signal Enhancement (IWAENC), Xi' an, Chiana, Sept. 2016.

[9] P. Shah, I. Lewis, S. Grant, and S. Angrignon, "Nonlinear acoustic echo cancellation using voltage and current feedback," IEEE/ACM Trans. Audio, Speech, Language Process., vol. 23, no. 10, pp. 1589-1599, 2015.

[10] L. A. Azpicueta-Ruiz, M. Zeller, A. R. Figueiras-Vidal, W. Kellermann, and J. Arenas-García, "Enhanced adaptive Volterra filtering by automatic attenuation of memory regions and its application to acoustic echo cancellation," IEEE Trans. Signal Process., vol. 61, no. 11, pp. 27452750, June 2013.

[11] J. Park and J.-H. Chang, "Frequency-domain Volterra filter based on data-driven soft decision for nonlinear acoustic echo suppression," IEEE Signal Process. Lett., vol. 21, no. 9, pp. 1088-1092, Sept. 2014.

[12] J. M. Gil-Cacho, M. Signoretto, T. Van Waterschoot, M. Moonen, and S. H. Jensen, "Nonlinear acoustic echo cancellation based on a slidingwindow leaky kernel affine projection algorithm," IEEE Trans. Audio, Speech, Language Process., vol. 21, no. 9, pp. 1867-1878, Sept. 2013.
[13] S. Van Vaerenbergh, L. A. Azpicueta-Ruiz, and D. Comminiello, "A split kernel adaptive filtering architecture for nonlinear acoustic echo cancellation," in 24th Eur. Signal Process. Conf. (EUSIPCO), Budapest, Hungary, Aug. 2016, pp. 1768-1772.

[14] M. Scarpiniti, D. Comminiello, R. Parisi, and A. Uncini, "Comparison of Hammerstein and Wiener systems for nonlinear acoustic echo cancelers in reverberant environments," in 17th Int. Conf. Digital Signal Process. (DSP), Corfù, Greece, July 2011, pp. 1-6.

[15] C. Hofmann, M. Guenther, C. Huemmer, and W. Kellermann, "Efficient nonlinear acoustic echo cancellation by partitioned-block significanceaware Hammerstein group models," in 24th Eur. Signal Process. Conf. (EUSIPCO), Budapest, Hungary, Aug. 2016, pp. 1783-1787.

[16] D. Comminiello, L. A. Azpicueta-Ruiz, M. Scarpiniti, A. Uncini, and J. Arenas-García, "Functional link based architectures for nonlinear acoustic echo cancellation," in Joint Workshop Hands-free Speech Commun. and Microphone Arrays (HSCMA), Edinburgh, UK, May 2011, pp. 180-184.

[17] G. L. Sicuranza and A. Carini, "A generalized FLANN filter for nonlinear active noise control," IEEE Trans. Audio, Speech, Language Process., vol. 19, no. 8, pp. 2412-2417, Nov. 2011.

[18] J. Benesty, T. Gänsler, D. R. Morgan, M. M. Sondhi, and S. L. Gay, Advances in Network and Acoustic Echo Cancellation, Digital Signal Processing. Springer-Verlag Berlin Heidelberg, 2001.

[19] S. L. Gay, "Dynamically regularized fast RLS with application to echo cancellation," in IEEE Int. Conf. Acoust., Speech and Signal Process. (ICASSP), Atlanta, GA, May 1996, vol. 2, pp. 957-960.

[20] Z. Chen, S. L. Gay, and S. Haykin, Least-Mean-Square Adaptive Filters, chapter Proportionate Adaptation: New Paradigms in Adaptive Filtering, pp. 293-334, John Wiley \& Sons, Inc., 2003.

[21] T. van Waterschoot, G. Rombouts, and M. Moonen, "Optimally regularized adaptive filtering algorithms for room acoustic signal enhancement," Signal Process., vol. 88, no. 3, pp. 594-611, Mar. 2008.

[22] I. Kodrasi, S. Goetze, and S. Doclo, "Increasing the robustness of acoustic multichannel equalization by means of regularization," in International Workshop on Acoustic Signal Enhancement (IWAENC), Aachen, Germany, Sept. 2012, pp. 1-4.

[23] D. Comminiello, M. Scarpiniti, L. A. Azpicueta-Ruiz, J. Arenas-García, and A. Uncini, "Nonlinear acoustic echo cancellation based on sparse functional link representations," IEEE Trans. Audio, Speech, Language Process., vol. 7, no. 22, pp. 1172-1183, July 2014.

[24] D. Comminiello, S. Scardapane, M. Scarpiniti, R. Parisi, and A. Uncini, "Online selection of functional links for nonlinear system identification," in Advances in Neural Networks: Computational and Theoretical Issues, S. Bassis, A. Esposito, and F. C. Morabito, Eds., vol. 37 of Smart Innovation, Systems and Technologies, pp. 39-47. Springer International Publishing AG, 2015.

[25] D. Comminiello, M. Scarpiniti, L. A. Azpicueta-Ruiz, J. Arenas-García, and A. Uncini, "A nonlinear architecture involving a combination of proportionate functional link adaptive filters," in 23rd Eur. Signal Process. Conf. (EUSIPCO), Nice, France, Sept. 2015, pp. 2919-2923.

[26] D. Comminiello, M. Scarpiniti, L. A. Azpicueta-Ruiz, J. Arenas-García, and A. Uncini, "A block-based combined scheme exploiting sparsity in nonlinear acoustic echo cancellation," in IEEE Int. Workshop on Machine Learning for Signal Process. (MLSP), Vietri Sul Mare, Italy, Sept. 2016.

[27] D. Comminiello, M. Scarpiniti, L. A. Azpicueta-Ruiz, J. Arenas-García, and A. Uncini, "Combined nonlinear filtering architectures involving sparse functional link adaptive filters," Signal Process., vol. 135, pp. 168-178, June 2017.

[28] A. H. Sayed, Adaptive Filters, John Wiley \& Sons, Hoboken, NJ, 2008.

[29] A. Uncini, Fundamentals of Adaptive Signal Processing, Signal and Communication Technology. Springer International Publishing AG, Cham, Switzerland, 2015, ISBN 978-3-319-02806-4.

[30] J. Benesty and S. L. Gay, "An improved PNLMS algorithm," in IEEE Int. Conf. Acoust., Speech and Signal Process. (ICASSP), Orlando, FL, May 2002, vol. 2, pp. 1881-1884.

[31] P. A. Naylor, J. Cui, and M. Brookes, "Adaptive algorithms for sparse echo cancellation," Signal Process., vol. 86, no. 6, pp. 1182-1192, June 2006.

[32] Y. Huang, J. Benesty, and J. Chen, Acoustic MIMO Signal Processing, Signals and Communication Technology. Springer-Verlag Berlin Heidelberg, 2006. 